\title{
Magnetoresistance in organic semiconductors
}

To the editor: $\mathrm{Hu}$ and $\mathrm{Wu}$ recently reported tunable magnetoresistance (MR) in organic semiconductors ${ }^{1}$, claiming that the geometry of multilayer diodes controls the relative influence of (electron-hole) polaron-pair (PP) dissociation and recombination under magnetic fields $(B)$. The underlying hypothesis is $B$-dependent intersystem crossing (ISC), conversion between spin manifolds of intermolecular exciton precursor PPs or (intramolecular) excitons. Hu and Wu postulate the generation of conductivity-influencing secondary polarons ${ }^{1}$, so that MR becomes sensitive to the electron-hole balance, determining the exciton-charge ratio ${ }^{1}$.

ISC is a relaxation process, which $\mathrm{Hu}$ and Wu claim to be controlled by external and internal Zeeman splitting, and singlet-triplet splitting ${ }^{1}$. These are effects usually described by the $B$-dependent Zeeman term and spin-spin interaction (spin-dipolar and exchange coupling). We agree that $B$ may control the singlettriplet content of electron-hole pairs ${ }^{2}$; the relevance to relaxation processes (for example, ISC) is not obvious. Spin-spin interactions of a spin-pair Hamiltonian do not determine relaxation directly. ISC must instead be described by spin-relaxation theory ${ }^{3}$, adding a fluctuation Hamiltonian $H_{\mathrm{f}}$ to a pair Hamiltonian $H_{0}$. $H_{\mathrm{f}}$ determines statistical addends to the Liouville equation (the Redfield matrix ${ }^{4}$ ) for the spin ensemble, and accounts for hyperfine interactions from randomly polarized and spatially distributed $\mathrm{H}$-atoms. $\mathrm{Hu}$ and $\mathrm{Wu}$ do not explain how these interactions and thus ISC are influenced by $B$. Can $B$ even change the PP singlet-triplet content to account for MR?

We could not infer values quoted for the internal Zeeman effect ("a range of $1-10 \mu \mathrm{eV}^{\prime \prime}$ ) from ref. 26 , which states zero-field splitting parameters $E$ and $D$ as $\sim 1 \mu \mathrm{eV}$ and $\sim 10 \mu \mathrm{eV}$, respectively, for naphthalene crystals. Suffice to note that the zero-field parameters in most organic materials are not determined by spin-orbital coupling as claimed by $\mathrm{Hu}$ and $\mathrm{Wu}$, but by spin-dipolar coupling ${ }^{4}$ within the PPs. Although measurements of the dipolar coupling for poly(phenylenevinylene $)^{5}(D \approx 72 \mathrm{mT}$, $E<10 \mathrm{mT}$ ) suggest that the material used by $\mathrm{Hu}$ and $\mathrm{Wu}$, poly[2-methoxy-5(2'-ethylhexyloxy)-1,4-phenylenevinylene], has similar parameters to naphthalene, it is unclear why the magnetic field should change spin-dependent $\mathrm{PP}$ recombination and dissociation rates. Following $\mathrm{Hu}$ and
$\mathrm{Wu}$, we consider PPs responsible for MR in terms of a two-spin system $(s=1 / 2)$ with Landé-factors $g_{\mathrm{a}}$ and $g_{\mathrm{b}}(\sim 2$ due to weak spin-orbit coupling), a mutual exchange coupling $J$, and a dipolar coupling determined by the zero-field matrix related to the zero-field parameters ${ }^{4}$. At low fields ( $\mu_{\mathrm{B}} B \approx E<D$, with $\mu_{\mathrm{B}}$ Bohr's magneton), $B / E$ controls mixing of the two triplet states $\left|T_{+}\right\rangle=|\uparrow \uparrow\rangle,\left|T_{-}\right\rangle=|\downarrow \downarrow\rangle ; B$ does not modify spin-dependent transition rates $^{4}$. For $\mu_{\mathrm{B}} B \geq D$ the spin-dipolar interaction is expressed in the high-field approximation $^{6}$, revealing a magneticfield-induced decoupling of the pair partners with the four PP eigenstates $|1\rangle=|\uparrow \uparrow\rangle,|2\rangle=\cos \phi|\uparrow \downarrow\rangle+\sin \phi|\downarrow \uparrow\rangle$, $|3\rangle=-\sin \phi|\uparrow \downarrow\rangle+\cos \phi|\downarrow \uparrow\rangle$ and $|4\rangle=\left|T_{-}\right\rangle=|\downarrow \downarrow\rangle ;|2\rangle$ and $|3\rangle$ are linear combinations of the product states $(|\uparrow \downarrow\rangle$ and $|\downarrow \uparrow\rangle)$, determined by the $B$-induced tilt angle

$$
\begin{aligned}
\phi & =\frac{1}{2} \arcsin \frac{(2 J+d)}{\sqrt{(2 J+d)^{2}+\left(\mu_{\mathrm{B}} B\left[g_{\mathrm{a}}-g_{\mathrm{b}}\right]\right)^{2}}} \\
& \equiv \frac{1}{2} \arcsin \frac{1}{\sqrt{1+\eta^{2}}}
\end{aligned}
$$

with $d=1 / 3 D\left(3 \cos ^{2} \Theta-1\right)$ describing the dipolar coupling' ${ }^{2}$ dependence on the spinpair's main axis angle $\Theta$ relative to the orientation of $B$. The angle $\phi$ depends on $\eta=\mu_{\mathrm{B}} B\left(g_{\mathrm{a}}-g_{\mathrm{b}}\right) /(2 J+d)$, the ratio of the difference of polaron Zeeman energies to the spin-spin coupling strengths. Contradicting $\mathrm{Hu}$ and $\mathrm{Wu}^{1}$, the PP singlettriplet ratio cannot change as the Zeeman splitting exceeds $J$ and $d$. Given this tiny value $\left(\mu_{\mathrm{B}} B\left(g_{\mathrm{a}}-g_{\mathrm{b}}\right)<200 \mathrm{neV}\right.$ at $B=328 \mathrm{mT}$; ref. 5), the crossover $(\eta \approx 1))$ occurs at $B \gtrsim 3 \mathrm{~T}$, far exceeding $\mathrm{Hu}$ and Wu's fields ${ }^{1}$. This $B$-limit is a lower estimate as it is likely that $|J| \gg|D|$ (ref. 5). Although equation (1) explains a $B$-dependence of an individual PP's singlet content, it cannot apply directly to disordered PP ensembles where all four eigenstates are generated equally. The singlet character of $|2\rangle$ increases with increasing $B$, that of $|3\rangle$ decreases; why should spin-dependent PP dissociation rates (which determine conductivity) then depend on $B$ ?

Testing for $B$-dependent singlet-triplet exciton formation requires simultaneous probing of singlet and triplet densities in organic light-emitting diodes. This is achieved by monitoring sensitizzed intrinsic phosphorescence ${ }^{7}$ (note that phosphorescent acceptor dopants modify triplet generation and $B$-dependencies ${ }^{8}$, as do field-dependent bimolecular reactions ${ }^{9}$ ).
Despite MR, strong fields (8 T) do not alter the singlet-triplet balance ${ }^{7}$, in agreement with triplet absorption spectroscopy ${ }^{10}$. Even at low fields (hyperfine field ${ }^{11}$ ) where MR is largest ${ }^{12}$, no change occurs. Although hyperfine interactions may be causally linked to MR (ref. 12), electron-nuclear spin coupling does not modify PP spin configurations. A viable alternative to $\mathrm{Hu}$ and Wu's excitonic picture ${ }^{1}$ (and to earlier hyperfine field-mixing models of fielddependent photoconductivity ${ }^{11}$ ) lies in the assumption of spin-dependent bipolaronic transport, providing a minimalistic parametric framework to describe positive and negative $\mathrm{MR}^{12}$.

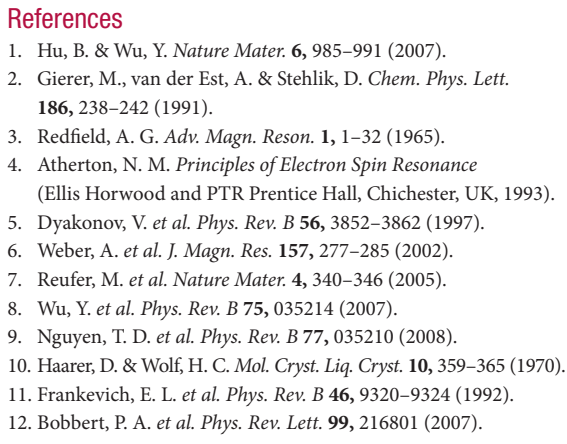

John M. Lupton and Christoph Boehme Department of Physics, University of Utah, 115 South 1400 East, Salt Lake City, Utah 84112, USA e-mail:lupton@physics.utah.edu;

boehme@physics.utah.edu

Hu and Wu reply: First, we have to clarify that in our work ${ }^{1}$ the different dissociation and charge reaction rates (not the recombination rates, as Lupton and Boehme questioned) between singlets and triplets are responsible for the magnetic-field-dependent generation of secondary charge carriers and of injection current. The different contributions to the dissociation and charge reaction from singlets and triplets have been shown by both theoretical calculations and experimental results ${ }^{2,3}$.

Second, Lupton and Boehme agree that an external magnetic field can change the singlet/triplet ratio, but argue that the change in singlet/triplet ratio with magnetic field does not come from intersystem crossing in polaron-pair states. However, the magnetic-field-induced modification of the singlet/triplet ratio through intersystem crossing has been both theoretically and experimentally predicted in electroluminescence ${ }^{4,5}$, photochemical reactions ${ }^{6}$ and photocurrents ${ }^{7}$. Lupton et al. reported that an external magnetic field 
increases both singlet fluorescence and triplet phosphorescence in the laddertype poly( $p$-phenylene), and consequently concluded that the singlet/triplet balance cannot be changed by an external magnetic field ${ }^{8}$. On the contrary, we recently found that monitoring both fluorescence and phosphorescence dependencies of magnetic field is an invalid method to claim whether or not an external magnetic field can change the singlet/triplet ratio if an internal energy transfer occurs ${ }^{9}$. In particular, a magnetic-field-induced increase of singlets at the exciting location can be seen as an increase of triplets at the emitting location if the energy transfer occurs from the exciting to the emitting location, and if the intersystem crossing is significant at the emitting location. This internal energy transfer together with intersystem crossing essentially results in an increase in both fluorescence and phosphorescence with increasing magnetic field. Furthermore, based on the hypothesis in which magneticfield-induced modification of intersystem crossing yields an increase in photocurrent, we observed that this increase disappears when the polaron pairs are removed by using the donor-acceptor interaction in organic solar cells ${ }^{10}$. This result clearly suggests that the magnetic-field effects contain the contribution from the intersystem crossing after the polaron pairs have formed. Although our experimental results of magnetic-field-dependent electroluminescence and photocurrent do not discuss how an external magnetic field changes the formation rates of singlet and triplet polaron pairs, they firmly imply that the intersystem crossing in polaron-pair states contributes to the magnetic-field-induced modification of the singlet/triplet ratio.

Third, with regards to the two-spin system of polaron pairs and the prediction that the intersystem crossover occurs at the magnetic field of about $3 \mathrm{~T}$, we notice that Lupton and Boehme's theoretical prediction is in conflict with experimental prediction that a low magnetic field can change the intersystem in weak-spinorbital-coupling materials with aromatic structures ${ }^{11}$. We argue that apart from affecting the access to the singlet-triplet energy, an external magnetic field also affects the mechanism for the flipping of the spin polarization, and the latter should also be considered in the discussion of magnetic-field effects. In our work ${ }^{1}$ we demonstrated experimentally that the excited-states-related magnetoresistance can be tuned between positive and negative values by adjusting the dissociation and charge reaction in the generation of secondary charge carriers through changing the balancing degree of bipolar charge injection. We believe that our experimental demonstration can mechanistically reveal how magnetic-field effects of singlet and triplet excited states are involved in the magnetoresistance in organic semiconductors.

References

1. Hu, B. \& Wu, Y. Nature Mater. 6, 985-991 (2007).

2. Kalinowski, J., Szmytkowski, J. \& Stampor, W. Chem. Phys. Lett. 378, 380-387 (2003).

3. Wohlgenannt, M. \& Vardeny, Z. V. J. Phys. Condens. Matter 15, R83-R107 (2003).

4. Kalinowski, J., Cocchi, M., Virgili, D., Di Marco, P. \& Fattori, V. Chem. Phys. Lett. 380, 710-715 (2003).

5. Gäraitz, C., Mückl, A. G. \& Cölle, M. J. Appl. Phys. 98, 104507 (2005).

6. Canfield, J. M., Belford, R. L., Debrunner, P. G. \& Schulten, K. J. Chem. Phys. 182, 1-18 (1994).

7. Ito, F., Ikoma, T., Akiyama, K., Watanabe, A. \& Tero-Kubota, S. J. Phys. Chem. B 109, 8707-8717 (2005).

8. Reufer, M. et al. Nature Mater. 4, 340-346 (2005).

9. Wu, Y., Xu, Z., Hu, B. \& Howe, J. Phys. Rev. B 75, 035214 (2007)

10. Xu, Z., Hu, B. \& Howe, J. J. Appl. Phys. 103, 043909 (2008).

11. Haarer, D. \& Wolf, H. C. Mol. Cryst. Liq. Cryst. 10, 359-380 (1970).

\section{$\mathrm{Bin} \mathrm{Hu}^{\star}$ and Yue $\mathrm{Wu}$}

Department of Materials Science and Engineering, University of Tennessee, Knoxville, Tennessee 37996, USA

*e-mail:bhu@utk.edu 